\title{
Tracking Data Certification for the Lunar Reconnaissance Orbiter
}

\author{
Patrick J. Morinelli ${ }^{1}$ \\ Honeywell Technology Solutions, Inc., Columbia, MD, 21046 \\ Joseph Socoby ${ }^{2}$ and Steve Hendry ${ }^{2}$ \\ Honeywell Technology Solutions, Inc., Columbia, MD, 21046 \\ and \\ Richard Campion ${ }^{2}$ \\ Honeywell Technology Solutions, Inc., Columbia, MD, 21046
}

\begin{abstract}
This paper details the National Aeronautics and Space Administration (NASA) Goddard Space Flight Center (GSFC) Flight Dynamics Facility (FDF) tracking data certification effort of the Lunar Reconnaissance Orbiter (LRO) Space Communications Network (SCN) complement of tracking stations consisting of the NASA White Sands 1 antenna (WS1), and the commercial provider Universal Space Network (USN) antennas at South Point, Hawaii; Dongara Australia; Weilheim, Germany; and Kiruna, Sweden. Certification assessment required the cooperation and coordination of parties not under the control of either the LRO project or ground stations as uplinks on cooperating spacecraft were necessary. The LRO range-tracking requirement of $10 \mathrm{~m} 1 \sigma$ could be satisfactorily demonstrated using any typical spacecraft capable of range tracking. Though typical Low Earth Orbiting (LEO) or Geosynchronous Earth Orbiting (GEO) spacecraft may be adequate for range certification, their measurement dynamics and noise would be unacceptable for proper Doppler certification of $1-3 \mathrm{~mm} / \mathrm{sec} 1 \sigma$. As LRO will orbit the Moon, it was imperative that a suitable target spacecraft be utilized which can closely mimic the expected lunar orbital Doppler dynamics of $+/-1.6 \mathrm{~km} / \mathrm{sec}$ and $+/-1.5 \mathrm{~m} / \mathrm{sec}^{2}$ to $+/-0.15 \mathrm{~m} / \mathrm{sec}^{2}$, is in view of the ground stations, supports coherent S-Band Doppler tracking measurements, and can be modeled by the FDF. In order to meet the LRO metric tracking data specifications, the SCN ground stations employed previously uncertified numerically controlled tracking receivers. Initial certification testing revealed certain characteristics of the units that required resolution before being granted certification.
\end{abstract}

\section{Nomenclature}

$\begin{array}{ll}A V G & =\text { Average } \\ B R T S & =\text { Bilateration Ranging Transponder System } \\ B P S K & =\text { Bi-Phase Shift Key } \\ F D F & =\text { Flight Dynamics Facility } \\ D S N & =\text { Deep Space Network } \\ F T P & =\text { File Transfer Protocol } \\ F O T & =\text { Flight Operations Team } \\ G E O & =\text { Geosynchronous Earth Orbiting } \\ G S F C & =\text { Goddard Space Flight Center } \\ G T D S & =\text { Goddard Trajectory Determination System } \\ I N P 2 & =\text { Internet Predict Version } 2\end{array}$

${ }^{1}$ Systems Engineer, NASA Goddard Space Flight Center Flight Dynamics Facility, NASA GSFC Mailstop 595.1, AIAA Member Grade MB

${ }^{2}$ Systems Engineer, NASA Goddard Space Flight Center Flight Dynamics Facility, NASA GSFC Mailstop 595.1, AIAA Member Grade N/A 


$\begin{array}{ll}J P L & =\text { Jet Propulsion Laboratory } \\ L E O & =\text { Low Earth Orbiting } \\ \text { LRO } & =\text { Lunar Reconnaissance Orbiter } \\ M A & =\text { Multiple Access Antenna } \\ M A C a l & =\text { Multiple Access Antenna Calibration } \\ N A S A & =\text { National Aeronautics and Space Administration } \\ N C O & =\text { Numerically Controlled Oscillator } \\ N M C & =\text { Network Management Center } \\ O-C & =\text { Observed Minus Computed } \\ R F I & =\text { Radio Frequency Interference } \\ S C N & =\text { Space Communications Network } \\ S T D & =\text { Standard Deviation } \\ S T G T & =\text { Second TDRS Ground Terminal } \\ T D R S & =\text { Tracking and Data Relay Satellite } \\ T H E M I S & =\text { Time History of Events and Macroscale Interactions during Substorms } \\ T T \& C & =\text { Tracking, Telemetry and Command } \\ U C B & =\text { University of California at Berkeley } \\ U T D F & =\text { Universal Tracking Data Format } \\ U S G S & =\text { United States Geological Survey } \\ U S N & =\text { Universal Space Network } \\ W S 1 & =\text { White Sands 1 Ground Station } \\ W S C & =\text { White Sands Complex } \\ W S G T & =\text { WSC Space to Ground Link Terminal }\end{array}$

\section{Introduction}

$\mathrm{T}$ HE primary objective of the National Aeronautics and Space Administration (NASA) Lunar Reconnaissance Orbiter (LRO) mission is to conduct investigations that support future human exploration of the Moon. In support of this goal, LRO will create the most comprehensive atlas of the Moon's features and resources, and as such high accuracy tracking data and orbit determination are necessary.

The LRO mission is supported by the LRO Space Communications Network (SCN) which is composed of three ground station networks. These networks include the Deep Space Network (DSN) operated by NASA Jet Propulsion Laboratory (JPL), the LRO network located at White Sands, New Mexico, and the commercial tracking service provider United Space Network (USN). These networks provide Tracking, Telemetry, and Commanding services to LRO for the life of the mission. The metric tracking data that is to be used by the NASA Goddard Space Flight Center's (GSFC) Flight Dynamics Facility (FDF) to estimate the LRO spacecraft orbit must be certified by the FDF to meet the network and mission requirements. This document details the NASA FDF tracking data certification effort of the LRO SCN complement of tracking stations consisting of the NASA White Sands 1 antenna (WS1), and the commercial provider Universal Space Network (USN) owned and operated antennae at South Point, Hawaii; and Dongara Australia; and collaborative sites Weilheim, Germany; and Kiruna, Sweden. The DSN stations support numerous missions where metric tracking data is used by the FDF for orbit and data validation purposes and therefore FDF certification is not necessary.

\section{Background}

The primary responsibility for successful ground station certification rests with the project or the ground station network seeking the certification. It is up to the project or ground station to schedule the needed resources, including satellite contacts, communications, and the FDF, to insure that FDF has sufficient information and tracking data to perform the evaluation and to respond to problems or questions regarding the tracking performance. Information needed by FDF includes the ground station antenna geodetic location, the antenna mount type and size, and a description of how the ground station sets certain data flags. FDF will provide the tracking data format, file naming conventions, and certain parameters needed by the ground station to correctly identify the tracking data, such as the antenna pad ID and the routing information. In addition, FDF will help the project or ground station network select suitable orbiting spacecraft to be used as targets for generating tracking data. It is critical that the selected target satellite be one for which the FDF can produce or obtain accurate orbits. It is these accurate orbits that FDF uses to make critical measurements related to the accuracy of the ground station metric tracking data. 
The NASA White Sands 1 antenna is located at the Second Tracking and Data Relay Satellite (TDRS) Ground Terminal (STGT) where it can be remotely operated from NASA GSFC.

The USN Network Management Center (NMC) is located in Horsham, Pennsylvania. For the LRO mission, USN uses two prime remote ground stations located at Dongara, Australia and Weilheim, Germany. USN maintains two backup stations to support the LRO mission; these stations are located at South Point, Hawaii and Kiruna, Sweden.

\section{Certification Requirements}

The SCN sites shall provide metric tracking data in the form of coherent S-Band Doppler and range. Although not explicitly specified as an LRO requirement, it is assumed that the range and Doppler accuracy specified is required of each data type collected individually as well as collected simultaneously with other measurement types. Note there are no LRO requirements for the certification of angle tracking data.

The WS1 antenna system and the USN antenna system shall provide range tracking data with accuracy of 10 meters $1 \sigma$. With direction from the LRO Project, the range accuracy requirement was interpreted as being individually applicable to both the residual mean and the residual standard deviation, but not their sum.

The WS1 antenna system shall provide Doppler tracking data with accuracy equal to or less than $1 \mathrm{~mm} / \mathrm{sec} 1 \sigma$, whereas the USN antenna system shall provide Doppler tracking data with accuracy equal to or less than $3 \mathrm{~mm} / \mathrm{sec}$ $1 \sigma$. With direction from the LRO Project, the Doppler accuracy requirement was interpreted as being applicable to the sum of the residual mean and standard deviation in conjunction with a sub-millimeter residual mean.

These requirements apply to measurements as received at FDF and include the effects of atmospheric refraction, random equipment biases, etc. A minimum of 80 percent of measurements in each pass shall be usable as indicated by the validity flags set by the tracker. Furthermore the sample rate for the range data shall be at least one measurement every 40 seconds and the Doppler measurements shall be performed over a 5 second integration period.

The tracking data shall be provided using the GSFC Universal Tracking Data Format (UTDF) ${ }^{3}$ and sent via file transfer protocol (FTP) to the FDF.

To achieve certification, each tracker must demonstrate range and 2-way Doppler tracking of an orbiting spacecraft to the specified accuracies. In order to provide sufficient data for meaningful statistics, 5 consecutive successful passes per tracker are required.

\section{Testing}

Each tracker has been certified individually, since some contributors to tracking errors, such as survey inaccuracies, antenna equipment, etc., are unique to each site. To achieve certification, each tracker demonstrated range and 2-way Doppler tracking to the specified accuracies noted above. Certification assessment required the cooperation and coordination of parties not under the control of either the LRO project or the SCN ground stations as uplinks on cooperating spacecraft were necessary. This cooperation and coordination was considerable for all parties involved. The evaluation method was to schedule passes on an available target spacecraft already being tracked by several certified operational trackers, interleaved with passes from the test sites. The target spacecraft must be suitable to FDF in that FDF must be able to generate or obtain an accurate orbit solution. An orbit solution determined from the operational trackers was used as a comparison "truth model" for evaluating the test sites' performance.

It was necessary that FDF be able to generate accurate orbits for the target spacecraft as it is these orbits that FDF uses to make critical measurements related to the accuracy of the SCN station metric tracking data. This necessitated that the amount of tracking of the chosen target spacecraft be increased and all tracking data be made available to the FDF.

The LRO range tracking requirement is such that it can be satisfactorily demonstrated using a typical low Earth orbiting (LEO) spacecraft. With cooperation from the United States Geological Survey (USGS), Landsat-5 was chosen as the target spacecraft with which to certify. However, the operational Landsat-5 orbit estimation was not sufficient to the level of accuracy necessary for certification of the levied requirements. Thus augmented tracking was requested to include range tracking data from ground stations located at Santiago, Chile; Merritt Island, Florida; and the NASA Space Network (SN) in addition to the standard Doppler data from Santiago; Poker Flat Facility, Alaska; and USGS Sioux Falls, South Dakota.

\footnotetext{
${ }^{3}$ Tracking and Acquisition Data Handbook for the Ground Network, GSFC, 453-HNDK-GN, January 2007
} 
Although suitable for the USN range tracking data certification, Landsat-5 unfortunately had radio frequency interference (RFI) issues at the White Sands Complex (WSC) that could not be mitigated as the Landsat-5 frequency is the same as the center frequency of the STGT Multiple Access (MA) antenna calibration service (Cal). Instead, the geosynchronous Earth orbiting (GEO) spacecraft TDRS-7 was utilized as the test target. The NASA SN granted permission for WS1 to test with TDRS-7 which was at the time a non-operational satellite of the TDRS fleet. Unlike operational TDRS whose orbits are determined from Bilateration Ranging Transponder System (BRTS) range and Doppler measurements, the TDRS-7 orbit was determined from the less accurate range tracking data collected from the WSC Space to Ground Link Terminal (WSGT) Tracking, Telemetry and Command (TT\&C) antenna during an intensive WS1 tracking campaign. Given that the orbit solution is fundamentally dependent upon the quality of the WSGT TT\&C range tracking data, it was necessary to verify its estimated bias. However, still being a highly restricted spacecraft, it was difficult to arrange for supplementary tracking to augment the TDRS-7 orbit solution accuracy. The WSGT TT\&C range bias was estimated 10 days later when TDRS-7 was tracked by DSN Goldstone and DSN Canberra. The FDF TDRS support group estimated the WSGT TT\&C bias to be $-27 \mathrm{~m}$ for the Primary Uplink / Primary Downlink equipment chain used during the period of the WS1 and DSN tracking.

Though LEO spacecraft may be adequate for range certification, their measurement dynamics and noise would be unacceptable for proper Doppler certification. As LRO will orbit the Moon, it was imperative that a suitable target spacecraft be utilized which can closely mimic the expected lunar orbital Doppler dynamics of $+/-1.6 \mathrm{~km} / \mathrm{sec}$ and $+/-1.5 \mathrm{~m} / \mathrm{sec}^{2}$ to $+/-0.15 \mathrm{~m} / \mathrm{sec}^{2}$, is in view of the stations being certified, supports coherent S-Band Doppler tracking measurements, and can be modeled by the FDF. Since there were no current NASA Lunar missions, the list of potential candidate target spacecraft is very brief. It is limited to those spacecraft with highly elliptical Earth orbits that for brief periods of time, while entering and exiting their perigee, emulate the orbital measurement dynamics of a Lunar orbit. The potential candidate target spacecraft list was quickly exhausted. The Polar spacecraft was considered as a potential candidate, particularly since it was capable of both range and Doppler tracking, but was unfortunately nearing its end of life and decommissioned prior to any certification opportunities. The only remaining spacecraft was that of the Time History of Events and Macroscale Interactions during Substorms (THEMIS) mission. With gracious cooperation from the University of California at Berkeley (UCB), THEMIS was the only, and thus vital, target spacecraft with which to certify the SCN Doppler tracking data. The THEMIS mission is comprised of five identical spacecraft, probes A through E, in varying orbital periods. Probes A, C, D, E were all considered satisfactory test targets. However, THEMIS-B, was discounted as it is in the largest orbit and not necessarily compensated for with additional tracking resulting in unsatisfactory orbit solution stability for certification.

Another LRO requirement, not directly related to metric tracking data accuracy, is that its supporting stations use the internet predict version 2 (INP2) predict format for LRO acquisition. Coincidentally, UCB already generated the INP2 predict format as a routine acquisition data product. FDF provided to UCB a script utility that would read an INP2 and identify view periods when the THEMIS orbital Doppler dynamics were within the range of expected lunar orbital Doppler dynamics with respect to the test site.

Operating procedures for UCB were to maximize high rate THEMIS data collection during perigee contacts where the space to ground link margin is the greatest. These high rate data collection passes are configured Bi-Phase Shift Key (BPSK) where the Doppler carrier is fully suppressed. Although the SCN stations are capable of reconstructing the Doppler carrier when BPSK is used, such opportunities were not considered for certification efforts as it was unsure how it may affect the Doppler measurement accuracy.

All five THEMIS spacecraft are spinning at $20 \mathrm{rpm}$ (3 sec spin period). A sinusoidal Doppler modulation on top of the regular Doppler profile is present when there is a displacement, lateral or in tilt angle, between the spacecraft body axis and the spin axis. This spin effect was observable in several of the THEMIS Doppler certification passes. However, this effect is very small and determined to be insignificant in regard to the levied accuracy requirement. The USN Doppler performance was sufficient to meet the LRO requirement specification without consideration of the spinning affect of the THEMIS probes. However, WS1 Doppler requirement was stringent enough that the spin effect had to be considered. A 5 second Doppler integration period was insufficient to properly model the spin, thus FDF requested that WS1 increase its Doppler sample rate to that of 1 second integration. This higher rate sampling provided a sufficient data population from which FDF could analytically remove the spin effect through a Fast Fourier Transform and then employ a moving average filter to reduce the sampling back to a 5 second integration to calculate the effective standard deviation.

Considering the proper Doppler dynamics, occurring only when the station had view of the THEMIS spacecraft when it entered or exited its perigee, nominal UCB BPSK operations and UCB and WS1 availability, the number of potential certification contacts was very limited. Additionally, the use of THEMIS introduced another RFI issue at WSC as its frequency also interfered with the STGT MA Cal service. Unlike the Landsat-5 RFI which could not be 
mitigated, that of THEMIS was alleviated by inhibiting the STGT MA Cal emitters and temporarily assigning the pseudo noise codes to the WSGT MA Cal service. It was necessary to inhibit the STGT MA Cal service for every THEMIS contact at WS1.

\section{Data Processing}

\section{A. Validation}

Validation of metric tracking data is the process of determining the quality of measurements from a tracker. For the USN metric tracking data the following validation criteria will apply. Gross anomalies and questionable data are noted and generally are not used for further orbit determination or calibration. Data that are free of anomalies are noted as being acceptable for further use in orbit determination, experiments, and calibration.

A tracking data pass is generally considered anomalous when 5 or more valid points and $20 \%$ of the valid data of one observation type (i.e., range or Doppler) are edited. Data flagged by the tracker as invalid are not considered anomalous. Other data that is not considered anomalous include data taken in masking regions, data below $7^{\circ}$ elevation for S-Band, bad data collected during the acquisition process, poor data caused by the spacecraft (spinning, antenna switching, antenna blockage, etc.), and data with problems caused in general by conditions not under the control of the ground station.

\section{B. Calibration}

Statistical performance values are derived from observed minus computed measurements (O-C) derived from weighted least-squares orbital solutions as generated by the FDF orbit determination and evaluation system Goddard Trajectory Determination System (GTDS). Gross outlier values are discarded followed by a standard sigma edit process. Each pass is examined to determine if a problem or anomaly prevents the pass data from being included in the calibrations analysis. The resulting $\mathrm{O}-\mathrm{C}$ values from the vetted passes for each measurement type being evaluated are compared to the accuracy specified in the requirement to determine if enough of the data collected meets the accuracy requirement.

\section{Results}

Although, preparations began much earlier, the certification campaign began with the first data collection on March 07, 2008 and concluded October 10, 2008. A high level of management was necessary to facilitate coordination of all of the entities involved, including the FDF, USN NMC and its tracking stations, WS1, NASA Ground and Space Networks and their respective scheduling offices, UCB and the THEMIS Flight Operations Team (FOT), USGS and the Landsat-5 FOT, and TDRSS.

All USN tracking data was delivered post pass from the USN NMC to the Flight Dynamics Product Center via FTP. All WS1 tracking data was delivered post pass to the FDF Communications Server via secure copy. The SCN has demonstrated the capability to provide comprehensive post pass UDTF files as well as contiguous files of five minute duration throughout tracking events.

\section{A. Engineering Results}

In order to meet the LRO metric tracking data specifications, it was necessary for the USN stations to upgrade from an IN-SNEC CORTEX NT to a CORTEX XL tracking receiver. All USN tracking data is collected in the native CORTEX format and forwarded to the USN NMC where it is compiled into UTDF. WS1 employed an RT Logic Telemetrix XL IF/Baseband (T70/70XL) Receive Range Command Processor tracking receiver. Although procured through different vendors, these tracking receivers are essentially identical in their processing of tracking measurements. Initial certification testing revealed certain characteristics of the new unit that required attention before continuing with certification.

The Doppler observation time tag was placed in the middle of the integration period rather than at the end, or time of the cycle counter reading, as defined for UTDF. The misplaced time tag resulted in a Doppler timing bias of one half of the integration period or sample rate.

Given the high accuracy LRO specifications, another improvement made to the Doppler processing was in determining the integer value of the UTDF Doppler phase count. The Doppler phase count, previously generated from a real number by truncation, was improved by rounding of the real number. 
The tracking receivers use a 32 bit numerically controlled oscillator (NCO) with a $200 \mathrm{MHz}$ reference. Thus the NCO resolution is $0.0466 \mathrm{~Hz}$ and is a source of a potential Doppler bias that was necessary to account for in the SCN tracking stations' frequency plans.

\section{B. Certification Results}

\section{USN Range Performance}

Formal range certification passes began on May 27, 2008, but did not complete until July 22, 2008 when all four USN stations completed five consecutive passes that successfully demonstrated the required LRO range accuracy requirement. The following tables summarize the results of the five successful and consecutive range certification passes for each of the four USN sites.

Table 1. USN Hawaii (USHS) Range Residual Certification Summary

\begin{tabular}{|c|c|c|c|c|c|}
\hline Target & Tracker & Date & Data & AVG & STD \\
\hline LANDSAT5 & USHS & 27-May & Range & $-1.4 \mathrm{~m}$ & $2.4 \mathrm{~m}$ \\
\hline LANDSAT5 & USHS & 2-Jun & Range & $-0.9 \mathrm{~m}$ & $3.3 \mathrm{~m}$ \\
\hline LANDSAT5 & USHS & 4-Jun & Range & $-5.6 \mathrm{~m}$ & $1.6 \mathrm{~m}$ \\
\hline LANDSAT5 & USHS & 6-Jun & Range & $-0.8 \mathrm{~m}$ & $1.9 \mathrm{~m}$ \\
\hline LANDSAT5 & USHS & 9-Jun & Range & $-4.0 \mathrm{~m}$ & $8.5 \mathrm{~m}$ \\
\hline
\end{tabular}

Table 2. USN Australia (USPS) Range Residual Certification Summary

\begin{tabular}{|c|c|c|c|c|c|}
\hline Target & Tracker & Date & Data & AVG & STD \\
\hline LANDSAT5 & USPS & 23-Jun & Range & $-0.3 \mathrm{~m}$ & $1.8 \mathrm{~m}$ \\
\hline LANDSAT5 & USPS & 25-Jun & Range & $+0.7 \mathrm{~m}$ & $2.2 \mathrm{~m}$ \\
\hline LANDSAT5 & USPS & 26-Jun & Range & $-5.7 \mathrm{~m}$ & $6.7 \mathrm{~m}$ \\
\hline LANDSAT5 & USPS & 27-Jun & Range & $-5.5 \mathrm{~m}$ & $5.9 \mathrm{~m}$ \\
\hline LANDSAT5 & USPS & 2-Jul & Range & $+1.8 \mathrm{~m}$ & $1.8 \mathrm{~m}$ \\
\hline
\end{tabular}

Table 3. USN Kiruna (KU1S) Range Residual Certification Summary

\begin{tabular}{|c|c|c|c|c|c|}
\hline Target & Tracker & Date & Data & AVG & STD \\
\hline LANDSAT5 & KU1S & 2-Jul & Range & $-6.3 \mathrm{~m}$ & $6.0 \mathrm{~m}$ \\
\hline LANDSAT5 & KU1S & 2-Jul & Range & $-6.2 \mathrm{~m}$ & $6.2 \mathrm{~m}$ \\
\hline LANDSAT5 & KU1S & 9-Jul & Range & $-7.0 \mathrm{~m}$ & $4.3 \mathrm{~m}$ \\
\hline LANDSAT5 & KU1S & 16-Jul & Range & $-6.7 \mathrm{~m}$ & $4.6 \mathrm{~m}$ \\
\hline LANDSAT5 & KU1S & 22-Jul & Range & $-6.6 \mathrm{~m}$ & $3.7 \mathrm{~m}$ \\
\hline
\end{tabular}

Table 4. USN Weilheim (WU2S) Range Residual Certification Summary

\begin{tabular}{|c|c|c|c|c|c|}
\hline Target & Tracker & Date & Data & AVG & STD \\
\hline LANDSAT5 & WU2S & 8-Jul & Range & $+4.5 \mathrm{~m}$ & $2.2 \mathrm{~m}$ \\
\hline LANDSAT5 & WU2S & 8-Jul & Range & $+0.2 \mathrm{~m}$ & $2.8 \mathrm{~m}$ \\
\hline LANDSAT5 & WU2S & 8-Jul & Range & $+2.6 \mathrm{~m}$ & $4.5 \mathrm{~m}$ \\
\hline LANDSAT5 & WU2S & $9-\mathrm{Jul}$ & Range & $-2.5 \mathrm{~m}$ & $4.9 \mathrm{~m}$ \\
\hline LANDSAT5 & WU2S & 9-Jul & Range & $+2.8 \mathrm{~m}$ & $3.4 \mathrm{~m}$ \\
\hline
\end{tabular}


Typical USN range residuals are as that of those shown below in Figure 1.

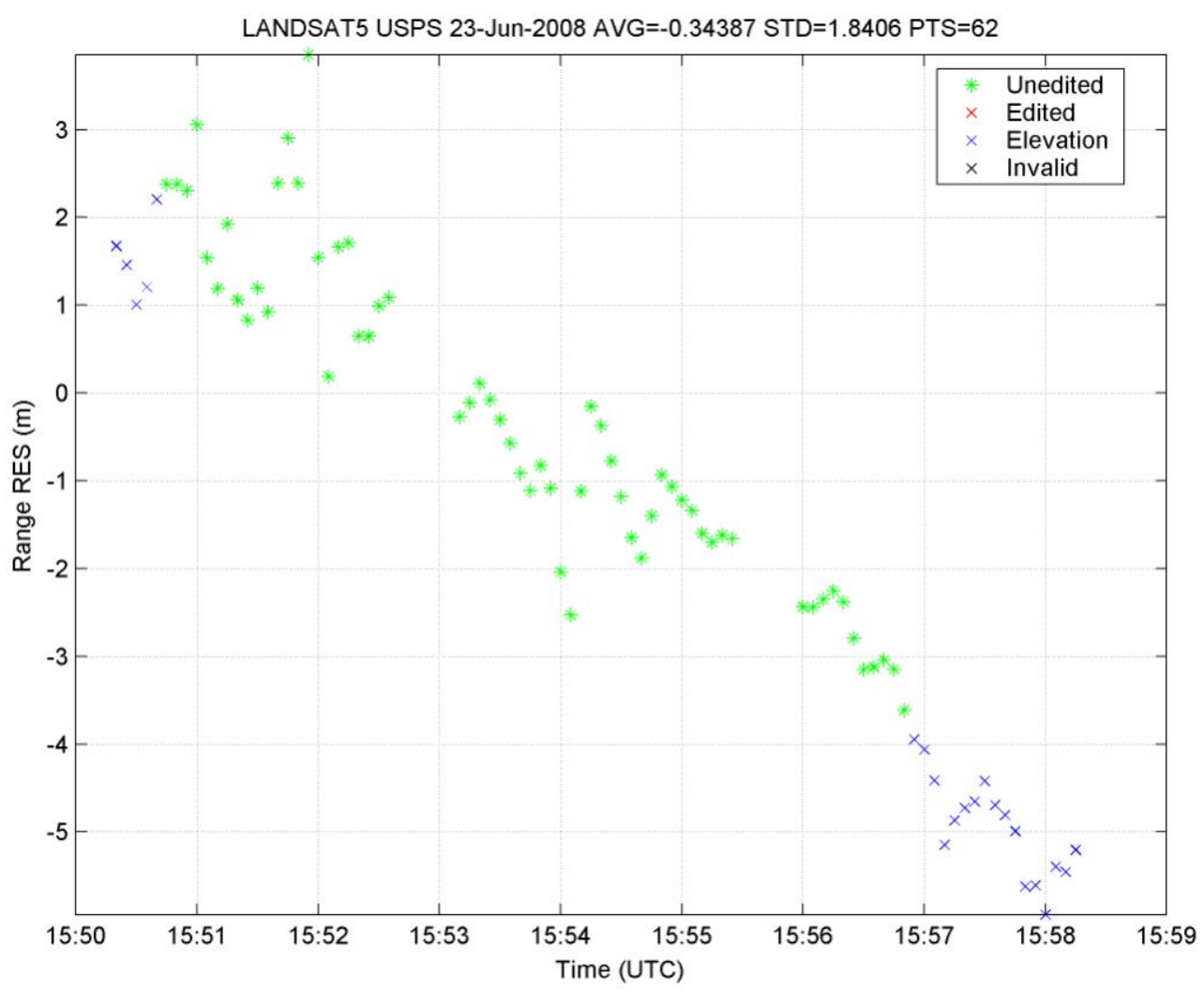

Figure 1 USN Range Residual Certification Plot 


\section{WS1 Range Performance}

Formal range certification passes began on March 14, 2008 and concluded March 15, 2008 when WS1 completed a total of nineteen distinct and consecutive passes successfully demonstrating the required LRO range accuracy requirement. The following table summarizes the results of the successful and consecutive range certification passes for WS1.

Table 5. WS1 Range Residual Certification Summary

\begin{tabular}{|c|c|c|c|c|}
\hline Target & $\begin{array}{c}\text { Date Time } \\
\text { (UTC) }\end{array}$ & Data & AVG & STD \\
\hline TDRS - 7 & 14-Mar 22:20 & Range & $-0.06 \mathrm{~m}$ & $0.46 \mathrm{~m}$ \\
\hline TDRS -7 & 14-Mar 23:00 & Range & $-1.89 \mathrm{~m}$ & $0.39 \mathrm{~m}$ \\
\hline TDRS -7 & 14-Mar 23:25 & Range & $-0.78 m$ & $1.08 \mathrm{~m}$ \\
\hline TDRS -7 & 15-Mar $00: 15$ & Range & $0.02 \mathrm{~m}$ & $0.47 \mathrm{~m}$ \\
\hline TDRS -7 & 15-Mar $00: 41$ & Range & $0.88 \mathrm{~m}$ & $0.16 \mathrm{~m}$ \\
\hline TDRS -7 & 15-Mar $00: 55$ & Range & $0.93 \mathrm{~m}$ & $0.21 \mathrm{~m}$ \\
\hline TDRS -7 & 15-Mar 01:13 & Range & $1.06 \mathrm{~m}$ & $0.11 \mathrm{~m}$ \\
\hline TDRS -7 & 15-Mar 02:05 & Range & $0.63 \mathrm{~m}$ & $0.17 \mathrm{~m}$ \\
\hline TDRS -7 & 15-Mar 02:25 & Range & $0.52 \mathrm{~m}$ & $0.16 \mathrm{~m}$ \\
\hline TDRS -7 & 15-Mar 16:09 & Range & $-0.25 m$ & $0.14 \mathrm{~m}$ \\
\hline TDRS -7 & 15-Mar $16: 24$ & Range & $-0.50 \mathrm{~m}$ & $0.11 \mathrm{~m}$ \\
\hline TDRS -7 & 15-Mar 16:40 & Range & $-0.63 m$ & $0.12 \mathrm{~m}$ \\
\hline TDRS -7 & 15-Mar 16:56 & Range & $-0.52 m$ & $0.11 \mathrm{~m}$ \\
\hline TDRS -7 & 15-Mar 17:10 & Range & $-0.26 m$ & $0.13 \mathrm{~m}$ \\
\hline TDRS -7 & 15-Mar $18: 20$ & Range & $0.09 \mathrm{~m}$ & $0.16 \mathrm{~m}$ \\
\hline TDRS -7 & 15-Mar $18: 35$ & Range & $-0.74 m$ & $0.32 \mathrm{~m}$ \\
\hline TDRS -7 & 15-Mar $18: 50$ & Range & $-0.56 m$ & $0.17 \mathrm{~m}$ \\
\hline TDRS -7 & 15-Mar 19:09 & Range & $-0.81 \mathrm{~m}$ & $0.12 \mathrm{~m}$ \\
\hline TDRS -7 & 15-Mar 19:25 & Range & $-0.82 m$ & $0.25 \mathrm{~m}$ \\
\hline
\end{tabular}


Typical WS1 range residuals are as that of those shown below in Figure 2.

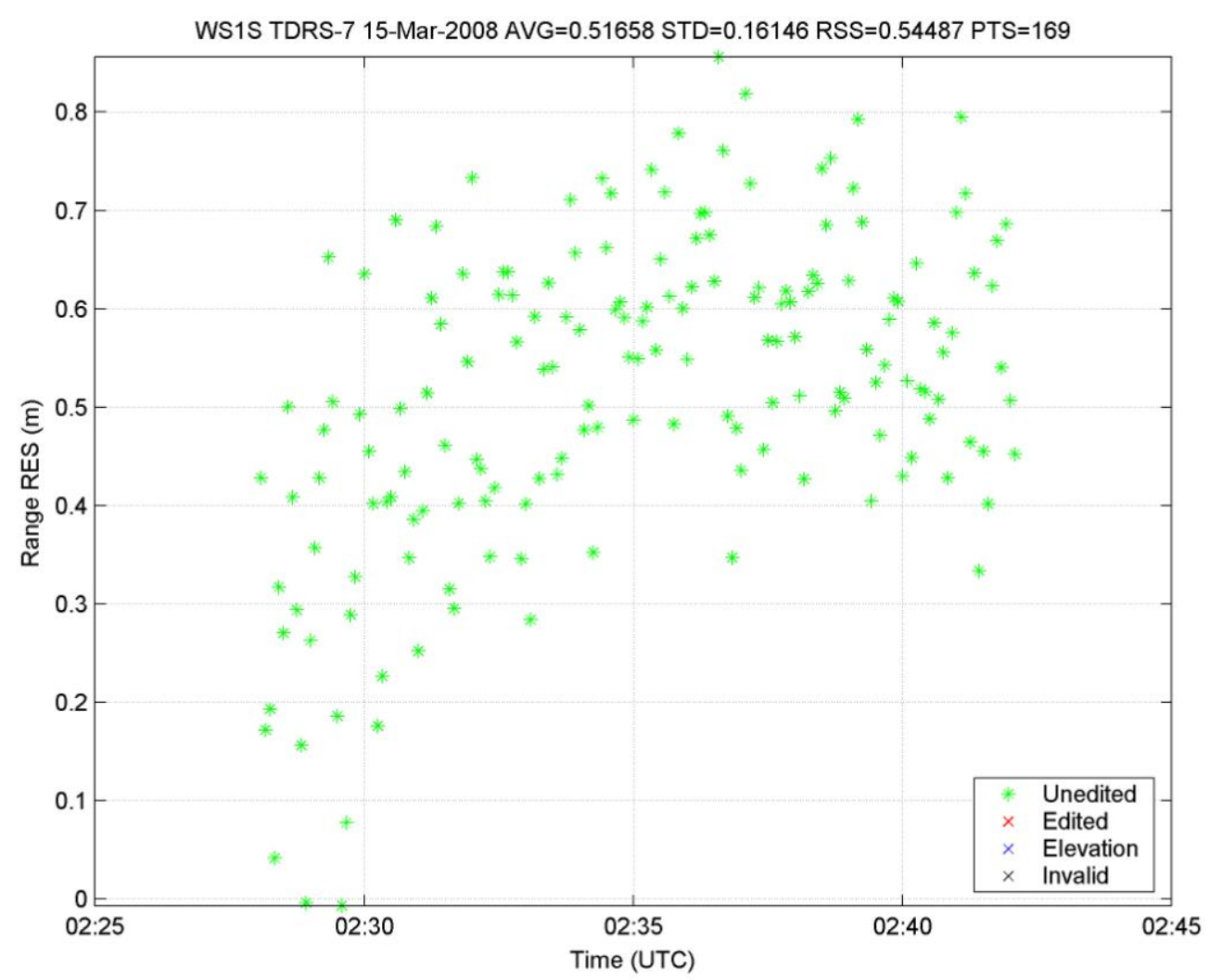

Figure 2 WS1 Range Residual Certification Plot

\section{USN Doppler Performance}

Formal Doppler certification passes began on June 23, 2008, but did not complete until July 25, 2008 when all four USN stations completed five consecutive passes that successfully demonstrated the required LRO Doppler accuracy requirement. The following tables summarize the results of the five successful and consecutive Doppler certification passes for each of the four USN sites.

Table 6. USN Hawaii (USHS) Doppler Residual Certification Summary

\begin{tabular}{|c|c|c|c|c|c|}
\hline Target & Tracker & Date & Data & AVG & STD \\
\hline THEMIS-D & USHS & 23-Jun & Doppler & $+0.10 \mathrm{~mm} / \mathrm{s}$ & $1.4 \mathrm{~mm} / \mathrm{s}$ \\
\hline THEMIS-D & USHS & 23-Jun & Doppler & $+0.10 \mathrm{~mm} / \mathrm{s}$ & $1.8 \mathrm{~mm} / \mathrm{s}$ \\
\hline THEMIS-E & USHS & 24-Jun & Doppler & $+0.15 \mathrm{~mm} / \mathrm{s}$ & $0.4 \mathrm{~mm} / \mathrm{s}$ \\
\hline THEMIS-D & USHS & 25-Jun & Doppler & $+0.15 \mathrm{~mm} / \mathrm{s}$ & $1.3 \mathrm{~mm} / \mathrm{s}$ \\
\hline THEMIS-E & USHS & 27-Jun & Doppler & $-0.07 \mathrm{~mm} / \mathrm{s}$ & $1.2 \mathrm{~mm} / \mathrm{s}$ \\
\hline
\end{tabular}


Table 7. USN Australia (USPS) Doppler Residual Certification Summary

\begin{tabular}{|c|c|c|c|c|c|}
\hline Target & Tracker & Date & Data & AVG & STD \\
\hline THEMIS-D & USPS & 23-Jun & Doppler & $+0.16 \mathrm{~mm} / \mathrm{s}$ & $2.2 \mathrm{~mm} / \mathrm{s}$ \\
\hline THEMIS-E & USPS & $25-J u n$ & Doppler & $+0.73 \mathrm{~mm} / \mathrm{s}$ & $1.7 \mathrm{~mm} / \mathrm{s}$ \\
\hline THEMIS-A & USPS & $27-J u n$ & Doppler & $+0.75 \mathrm{~mm} / \mathrm{s}$ & $1.6 \mathrm{~mm} / \mathrm{s}$ \\
\hline THEMIS-A & USPS & $12-J u l$ & Doppler & $+0.48 \mathrm{~mm} / \mathrm{s}$ & $1.7 \mathrm{~mm} / \mathrm{s}$ \\
\hline THEMIS-A & USPS & $13-J u l$ & Doppler & $+0.46 \mathrm{~mm} / \mathrm{s}$ & $1.9 \mathrm{~mm} / \mathrm{s}$ \\
\hline
\end{tabular}

Table 8. USN Kiruna (KU1S) Doppler Residual Certification Summary

\begin{tabular}{|c|c|c|c|c|c|}
\hline Target & Tracker & Date & Data & AVG & STD \\
\hline THEMIS-A & KU1S & 24-Jun & Doppler & $-0.28 \mathrm{~mm} / \mathrm{s}$ & $1.6 \mathrm{~mm} / \mathrm{s}$ \\
\hline THEMIS-A & KU1S & 24-Jun & Doppler & $-0.24 \mathrm{~mm} / \mathrm{s}$ & $1.8 \mathrm{~mm} / \mathrm{s}$ \\
\hline THEMIS-A & KU1S & 25-Jun & Doppler & $-0.31 \mathrm{~mm} / \mathrm{s}$ & $1.7 \mathrm{~mm} / \mathrm{s}$ \\
\hline THEMIS-A & KU1S & 25-Jun & Doppler & $-0.16 \mathrm{~mm} / \mathrm{s}$ & $1.5 \mathrm{~mm} / \mathrm{s}$ \\
\hline THEMIS-A & KU1S & 25-Jun & Doppler & $-0.10 \mathrm{~mm} / \mathrm{s}$ & $1.9 \mathrm{~mm} / \mathrm{s}$ \\
\hline
\end{tabular}

Table 9. USN Weilheim (WU2S) Doppler Residual Certification Summary

\begin{tabular}{|c|c|c|c|c|c|}
\hline Target & Tracker & Date & Data & AVG & STD \\
\hline THEMIS-C & WU2S & 24-Jun & Doppler & $+0.20 \mathrm{~mm} / \mathrm{s}$ & $1.3 \mathrm{~mm} / \mathrm{s}$ \\
\hline THEMIS-C & WU2S & $26-J u n$ & Doppler & $+0.91 \mathrm{~mm} / \mathrm{s}$ & $1.3 \mathrm{~mm} / \mathrm{s}$ \\
\hline THEMIS-D & WU2S & 23-Jul & Doppler & $-0.49 \mathrm{~mm} / \mathrm{s}$ & $1.4 \mathrm{~mm} / \mathrm{s}$ \\
\hline THEMIS-D & WU2S & $24-J u l$ & Doppler & $-0.42 \mathrm{~mm} / \mathrm{s}$ & $1.2 \mathrm{~mm} / \mathrm{s}$ \\
\hline THEMIS-D & WU2S & 25-Jul & Doppler & $-0.89 \mathrm{~mm} / \mathrm{s}$ & $1.3 \mathrm{~mm} / \mathrm{s}$ \\
\hline
\end{tabular}


Typical USN Doppler residuals are as that of those shown below in Figure 3.

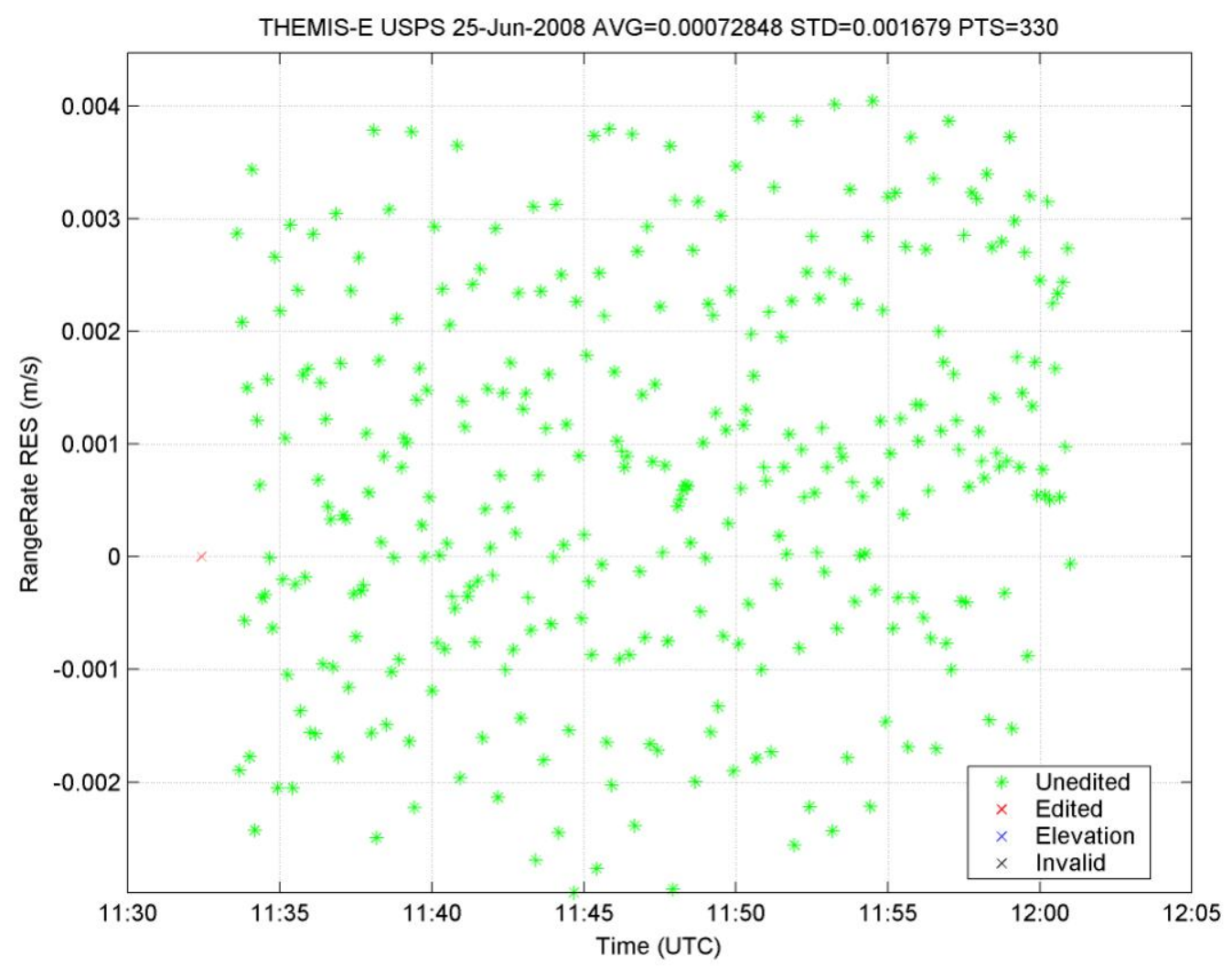

Figure 3 USN Doppler Residual Certification Plot

11

American Institute of Aeronautics and Astronautics 
As discussed in Section IV, it was imperative that a target spacecraft be utilized which can closely mimic the expected lunar orbital Doppler dynamics of $+/-1.6 \mathrm{~km} / \mathrm{sec}$ and $+/-1.5 \mathrm{~m} / \mathrm{sec}^{2}$ to $+/-0.15 \mathrm{~m} / \mathrm{sec}^{2}$. Shown below in Figure 4 is the accompanying Doppler dynamics of the pass data of Figure 3 and is representative of the target dynamics.

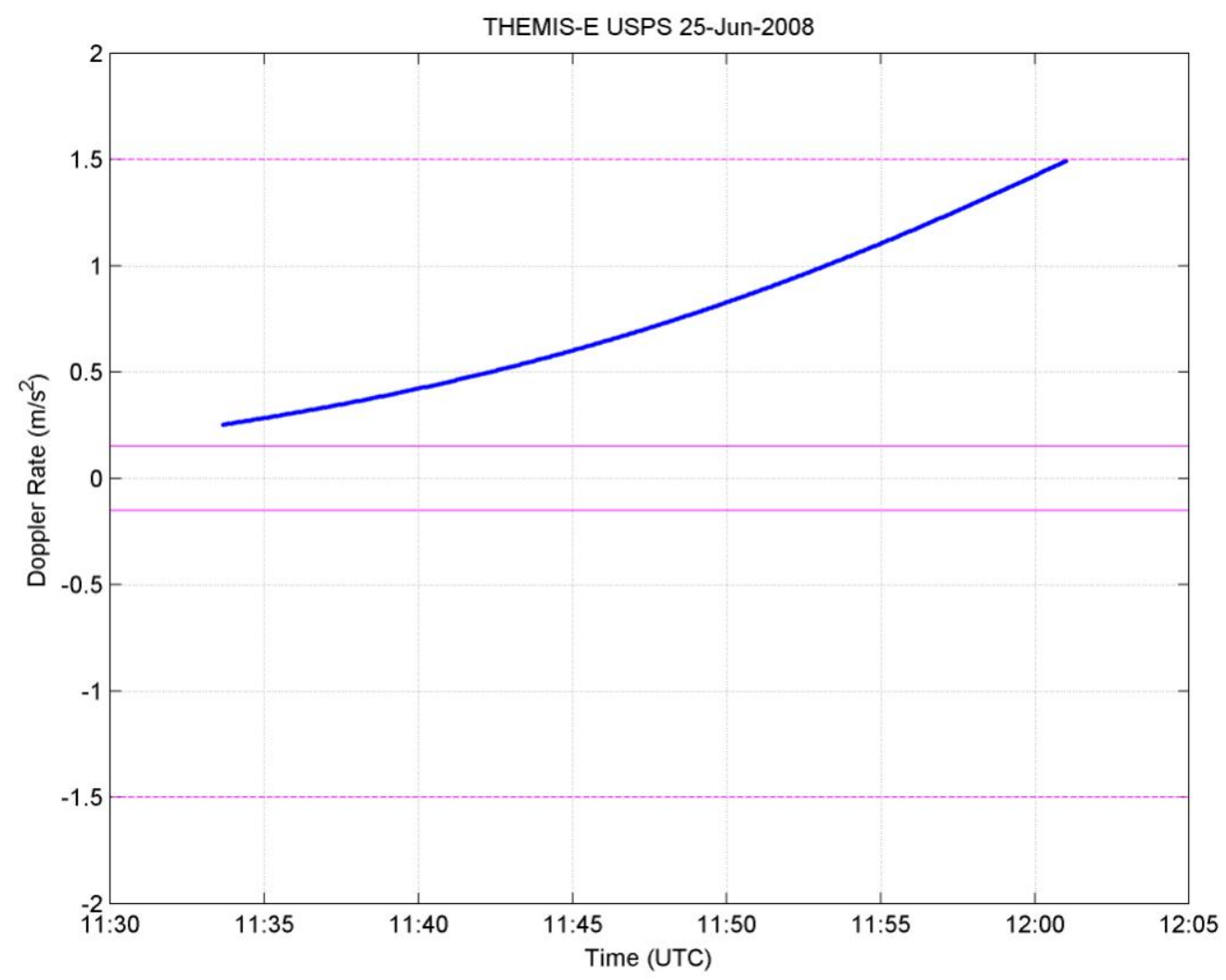

Figure 4 Doppler Rate Certification Plot

The solid lines at $+/-0.15 \mathrm{~m} / \mathrm{sec}^{2}$ represent the minimum Doppler rate allowable for certification while the dashed lines correspond to the expected maximum rate of $+/-1.5 \mathrm{~m} / \mathrm{sec}^{2}$ in lunar orbit. However, certification was not bound to the maximum rate as it is actually more challenging for a tracker to meet the accuracy specification at higher rates, hence the dashed boundary and potentiality of the tracking station to track beyond this rate at its own discretion. 
Shown below in Figure 5 is another Doppler residual certification plot that clearly reveals the THEMIS spacecraft spin through Doppler aliasing.

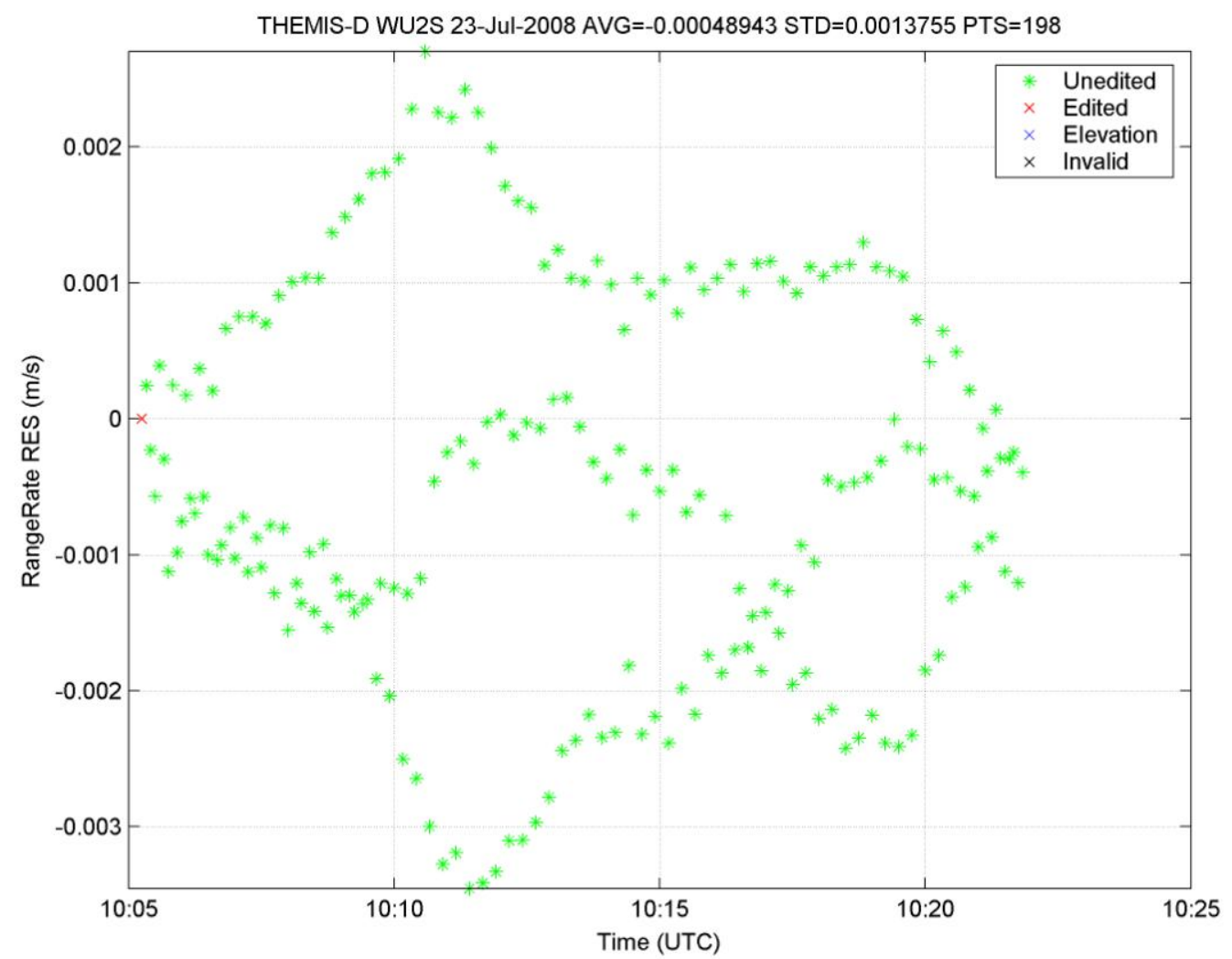

Figure 5 Spinning Spacecraft Doppler Aliasing Residuals

As is discussed in Section IV, the USN Doppler performance was sufficient to meet the LRO requirement specification of $3 \mathrm{~mm} / \mathrm{sec}$ without consideration of the spinning affect of the THEMIS probes. 


\section{WS1 Doppler Performance}

Formal Doppler certification passes began on September 26, 2008, and concluded October 10, 2008 when WS1 completed five consecutive passes that successfully demonstrated the required LRO Doppler accuracy requirement. The following table summarizes the results of the five successful and consecutive Doppler certification passes for WS1. As is described in Section IV, the results include the analytical removal of the spacecraft spin effect.

Table 10. WS1 Doppler Residual Certification Summary

\begin{tabular}{|c|c|c|c|c|c|c|}
\hline Target & Date & Data & AVG & STD & Comments & \\
\hline THEMIS-E & $26-$ Sep & Doppler & $-0.14 \mathrm{~mm} / \mathrm{s}$ & $6.8 \mathrm{~mm} / \mathrm{s}$ & s/c spin not & removed, 1/1sec \\
\hline THEMIS-E & $26-$ Sep & & & $0.34 \mathrm{~mm} / \mathrm{s}$ & s/c spin & removed, 1/1sec \\
\hline THEMIS-E & 26-Sep & Doppler & & $0.17 \mathrm{~mm} / \mathrm{s}$ & s/c spin & removed, $1 / 5 \mathrm{sec}$ \\
\hline THEMIS-E & $27-$ Sep & Doppler & $0.07 \mathrm{r}$ & $7.20 \mathrm{~mm} / \mathrm{s}$ & s/c spin not & removed, $1 / 1 \mathrm{sec}$ \\
\hline THEMIS-E & $27-\operatorname{Sep}$ & Doppler & & $0.40 \mathrm{~mm} / \mathrm{s}$ & s/c spin & removed, $1 / 1 \mathrm{sec}$ \\
\hline THEMIS-E & 27-Sep & & & $0.16 \mathrm{~mm} / \mathrm{s}$ & s/c spin & removed, \\
\hline THEMIS-E & $08-0 c t$ & Doppler & $0.08 \mathrm{~mm} / \mathrm{s}$ & $6.5 \mathrm{~mm} / \mathrm{s}$ & s/c spin not & removed, $1 / 1 \mathrm{sec}$ \\
\hline THEMIS-E & $08-0 c t$ & Doppler & & $0.44 \mathrm{~mm} / \mathrm{s}$ & s/c spin & removed, $1 / 1 \mathrm{sec}$ \\
\hline THEMIS-E & $08-0 \mathrm{ct}$ & Doppler & & $0.13 \mathrm{~mm} / \mathrm{s}$ & s/c spin & removed, \\
\hline THEMIS-E & 09-oct & Doppler & $-0.09 \mathrm{~mm} / \mathrm{s}$ & $7.6 \mathrm{~mm} / \mathrm{s}$ & s/c spin not & removed, \\
\hline THEMIS-E & 09-oct & Doppler & & $0.26 \mathrm{~mm} / \mathrm{s}$ & s/c spin & removed, $1 / 1 \mathrm{sec}$ \\
\hline THEMIS-E & 09-Oct & Doppler & & $0.07 \mathrm{~mm} / \mathrm{s}$ & s/c spin & removed, \\
\hline THEMIS-E & $10-$ oct & Doppler & $-0.07 \mathrm{~mm} / \mathrm{s}$ & $4.3 \mathrm{~mm} / \mathrm{s}$ & s/c spin not & removed, 1/1sec \\
\hline THEMIS-E & $10-$ oct & Doppler & & $0.32 \mathrm{~mm} / \mathrm{s}$ & s/c spin & removed, 1/1sec \\
\hline THEMIS-E & $10-$ oct & Doppler & & $0.12 \mathrm{~mm} / \mathrm{s}$ & s/c spin & removed, $1 / 5 \mathrm{sec}$ \\
\hline
\end{tabular}

The WS1 Doppler requirement was stringent enough that it could not be satisfied with the Doppler aliasing due to the THEMIS spacecraft spin. The 5 second Doppler integration specification was insufficient to adequately model the spin, thus FDF requested an increased sample rate to that of 1 second integration. This higher rate sampling provided a sufficient data population from which FDF could analytically remove the spin effect through a Fast Fourier Transform and then employ a moving average filter to reduce the sampling back to a 5 second integration to calculate the effective standard deviation. Typical WS1 Doppler residuals are as that of those shown below in Figure 6. 


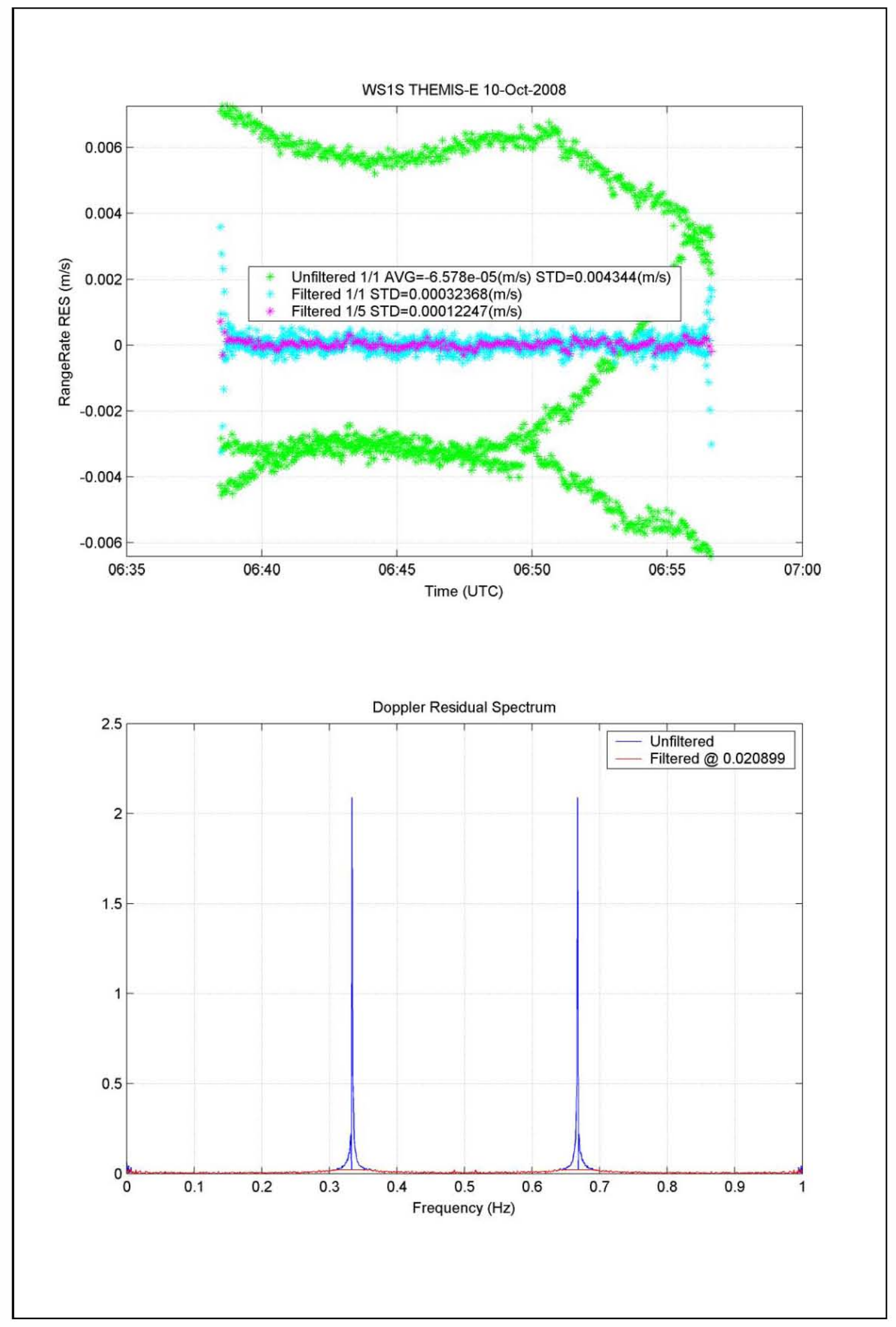

Figure 6. Fast Fourier Transformation of Aliased Doppler Residuals

15

American Institute of Aeronautics and Astronautics 
In this example, the green data points are that of the high rate 1/1sec Doppler residuals including the spacecraft spin aliasing. A Fast Fourier Transform reveals a Doppler residual frequency spectrum demonstrative of the THEMIS $20 \mathrm{rpm}$ spin rate with peaks at 0.33 and $0.66 \mathrm{~Hz}$ plotted in blue. In this spectrum the aliased Doppler data can be removed at these peaks. With the Doppler spin aliasing removed, plotted in red, the Fast Fourier Transformation is reversed and then reevaluated, plotted in cyan. A moving average filter is then used to return the data sampling back to the required $5 \mathrm{sec}$ integration, plotted in magenta, and shown to meet the $1 \mathrm{~mm} / \mathrm{sec}$ specification.

\section{WS1 Simultaneous Range and Doppler Performance}

As discussed in Section IV, there were no suitable test targets from which both range and Doppler tracking measurements could be collected simultaneously. As such WS1 range and Doppler metric tracking data were certified individually on separate target spacecraft. Following WS1's certification campaign, the opportunity to collect both range and Doppler tracking measurements simultaneously from one spacecraft was presented when THEMIS began its own certification of its spacecraft ranging operations in preparations for its follow on mission ARTEMIS: Acceleration, Reconnection, Turbulence, and Electrodynamics of Moon's Interaction with the Sun. WS1 was requested to assist in the effort of estimating the THEMIS onboard ranging transponder delay. This was a fortunate circumstance as it allowed simultaneous range and Doppler tracking measurements and also the possibility of alleviating the need for a separate test target in order to maintain range tracking certification proficiency.

Unfortunately, excessive Doppler noise was observed while WS1 was transmitting range tones. The Doppler degradation was significant and failed LRO specifications. The source of the degradation was traced to a software issue that had gone unnoticed due to the limitation of available test targets resulting in the constraint that range and Doppler tracking be certified individually.

The software issue was quickly corrected and WS1 demonstrated nominal Doppler performance to the LRO specifications while simultaneously transmitting range tones.

\section{Conclusion}

All four USN stations, Hawaii, Australia, Kiruna, and Weilheim, have each demonstrated five consecutive successful passes of range tracking using Landsat-5 and five each of Doppler tracking using THEMIS. All range and Doppler tracking data adequately satisfied the LRO requirement specification of 10 meters $1 \sigma$ and $3 \mathrm{~mm} / \mathrm{sec} 1 \sigma$, respectively, as stated in Section III.

The WS1 station has demonstrated five consecutive successful passes of range tracking using TDRS-7 and five of Doppler tracking using THEMIS. All range and Doppler tracking data adequately satisfied the LRO requirement specification of 10 meters $1 \sigma$ and $1 \mathrm{~mm} / \mathrm{sec} 1 \sigma$, respectively, as stated in Section III.

\section{Acknowledgments}

The authors of this paper wish to acknowledge UCB, USGS and TDRS for allowing the use of their spacecraft as targets of opportunity and for all of their generous assistance. Acknowledgment is also given to all the remaining parties involved, including the NASA Near Earth Network and SN, WS1, USN and FDF. 